\title{
RAPID COMPUTATION OF L-FUNCTIONS ATTACHED TO MAASS FORMS
}

\author{
ANDREW R. BOOKER AND HOLGER THEN
}

\begin{abstract}
Let $L$ be a degree- $2 L$-function associated to a Maass cusp form. We explore an algorithm that evaluates $t$ values of $L$ on the critical line in time $O\left(t^{1+\varepsilon}\right)$. We use this algorithm to rigorously compute an abundance of consecutive zeros and investigate their distribution.
\end{abstract}

\section{INTRODUCTION}

In 2, the first author presented an algorithm for the rigorous computation of $L$-functions associated to automorphic forms. The algorithm is efficient when one desires many values of a single $L$-function or values of many $L$-functions with a common $\Gamma$-factor. In this paper, we explore the prototypical case of a family of $L$-functions to which that does not apply, namely Maass cusp forms in the eigenvalue aspect.

As described in [2, §5], one of the main challenges when computing $L$-functions is the evaluation of the inverse Mellin transform of the associated $\Gamma$-factor. Rubinstein [16] describes an algorithm based on continued fractions that performs well in practice, but for which it seems to be very difficult to obtain rigorous error bounds. On the other hand, the algorithm in [2], following Dokchitser [7, uses a precomputation based on simpler power series expansions that are easy to make rigorous; it works well for motivic $L$-functions of low weight, but suffers from catastrophic precision loss when the shifts in the $\Gamma$-factor grow large, as is the case for Maass forms.

A well-known similar problem occurs when one attempts to evaluate an $L$-function high up in the critical strip. Rubinstein, following an idea of Lagarias and Odlyzko [12, has demonstrated that this can be dealt with effectively by multiplying by an exponential factor to compensate for the decay of the $\Gamma$-factor; specifically, for a complete $L$-function $\Lambda(s)$ of degree $d$, one works with $e^{-i \theta s} \Lambda(s)$ for a suitable $\theta<\pi d / 4$. This idea can be made to work for general $L$-functions, including those associated to Maass forms (albeit with the problems related to precision loss noted above, if the $\Gamma$-factor is not fixed), and Molin [13] has worked out rigorous numerical methods in quite wide generality.

For the specific case of Maass cusp forms, Vishe [19] (see also [8]) has shown that the "right" factor to multiply by to account for the variation in both $t$ and the $\Gamma$-factor shifts is not the exponential function $e^{-i \theta s}$, but rather the hypergeometric function

$$
{ }_{2} F_{1}\left(\frac{s+\epsilon+i r}{2}, \frac{s+\epsilon-i r}{2} ; \frac{1}{2}+\epsilon ;-\tan ^{2} \theta\right)
$$

where $\epsilon$ denotes the parity of the Maass form, and $\frac{1}{4}+r^{2}$ is its Laplacian eigenvalue. To understand the motivation for this factor, consider first the case of a classical holomorphic cuspform $f$, for which the $L$-function is defined via the Mellin transform

$$
\Lambda(s)=\int_{0}^{\infty} f(i y) y^{s} \frac{d y}{y} .
$$

Since $f$ is holomorphic and vanishes in the cusp, we can change the contour of integration from $(0, \infty)$ to $e^{i \theta}(0, \infty)$ for some $\theta \in(-\pi / 2, \pi / 2)$; writing $y=e^{i \theta} u$ for $u \in \mathbb{R}$, we obtain

$$
e^{-i \theta s} \Lambda(s)=\int_{0}^{\infty} f\left(i e^{i \theta} u\right) u^{s} \frac{d u}{u} .
$$

Date: October 15, 2018.

The authors wish to express their thanks to Andreas Strömbergsson and Pankaj Vishe for offering deep insight into their methods. H. T. thanks Brian Conrey, Dennis Hejhal, Jon Keating, Anton Mellit, and Franzesco Mezzadri for inspiring discussions. A. B. and H. T. acknowledge support from EPSRC grant EP/H005188/1. 
Thus, Rubinstein's exponential factor arises naturally from a contour rotation.

For a Maass form $f$ of weight 0 and even parity (say), we similarly have the integral representation

$$
\Lambda(s)=\int_{0}^{\infty} f(i y) y^{s-\frac{1}{2}} \frac{d y}{y} .
$$

Since $f$ is no longer holomorphic in this case, we cannot simply rotate the contour in this expression, but we are free to start with the rotated integral $\int_{0}^{\infty} f\left(i e^{i \theta} u\right) u^{s-\frac{1}{2}} \frac{d u}{u}$ and try to relate it back to $\Lambda(s)$. As we show in $\$ 2$, this can be done, and the two are related essentially by the factor (1.1).

We analyze this strategy in greater detail in $\$ 2$, but the upshot is that to compute Maass form $L$-functions for a wide range of values of $r$ and $t$, it suffices to compute $f\left(i e^{i \theta} u\right)$ for suitable values of $\theta$ and $u$. In turn, using modularity to move each point to the fundamental domain, the problem reduces to computing the $K$-Bessel function $K_{i r}(y)$ for various $r$ and $y$. Fortunately, that is a problem that underlies all computational aspects of Maass cusp forms and has been well studied; see, for instance, [3].

Numerical results. In $\$ 8$, using as input the rigorous numerical Maass form data of [1] we compute values of the corresponding Maass form $L$-functions and use the resulting numerical data to test conjectures about the distribution of zeros of Maass form $L$-functions in the $t$ - and $r$-aspects. In particular, we show that the phenomenon of zero repulsion around $\frac{1}{2} \pm i r$ that Strömbergsson observed [18] disappears in the large eigenvalue limit.

We derive rigorous error estimates and use the interval arithmetic package MPFI [15] throughout our computations to manage round-off errors. Thus, modulo bugs in the software or hardware, our numerical results are rigorous.

\section{Preliminaries on MaAss forms}

Let $f \in L^{2}\left(\Gamma_{1}(N) \backslash \mathbb{H}\right)$ be a cuspidal Maass newform and Hecke eigenform of weight 0 and level $N$. Then $f$ has a Fourier expansion of the form

$$
f(x+i y)=\sqrt{y} \sum_{n=1}^{\infty} a_{n} K_{i r}(2 \pi n y) \cos ^{(-\epsilon)}(2 \pi n x),
$$

where $a_{n}$ is the $n$th Hecke eigenvalue of $f, \frac{1}{4}+r^{2}$ is its eigenvalue for the hyperbolic Laplacian $-y^{2}\left(\frac{\partial^{2}}{\partial x^{2}}+\frac{\partial^{2}}{\partial y^{2}}\right), \epsilon \in\{0,1\}$ indicates the parity, and

$$
\cos ^{(-\epsilon)}(x):= \begin{cases}\cos x & \text { if } \epsilon=0 \\ \sin x & \text { if } \epsilon=1\end{cases}
$$

Moreover, $f$ is related to its dual $\bar{f}$ via the Fricke involution, so that

$$
f(z)=w \overline{\left(-\frac{1}{N z}\right)}
$$

for some complex number $w$ with $|w|=1$.

Associated to $f$ we have the $L$-function $L(s, f)$, defined for $\operatorname{Re}(s)>1$ by the series

$$
L(s, f):=\sum_{n=1}^{\infty} \frac{a_{n}}{n^{s}}
$$

It follows from 2.1 that $L(s, f)$ continues to an entire function and satisfies a functional equation relating its values at $s$ and $1-\bar{s}$. To see this, let ${ }_{2} F_{1}$ denote the Gauss hypergeometric function

$$
{ }_{2} F_{1}(\alpha, \beta ; \gamma ; z):=1+\sum_{n=1}^{\infty} \frac{(\alpha)_{n}(\beta)_{n} z^{n}}{(\gamma)_{n} n !}
$$


and consider the family of $\Gamma$-factors

$$
\begin{aligned}
\gamma_{\theta}(s, f):=i^{-\epsilon} w^{-1 / 2}\left(\frac{\cos \theta}{\sqrt{N}}\right)^{1 / 2-s} \Gamma_{\mathbb{R}}(s+\epsilon+i r) \Gamma_{\mathbb{R}}(s+\epsilon-i r) \\
\cdot{ }_{2} F_{1}\left(\frac{s+\epsilon+i r}{2}, \frac{s+\epsilon-i r}{2} ; \frac{1}{2}+\epsilon ;-\tan ^{2} \theta\right),
\end{aligned}
$$

where $\Gamma_{\mathbb{R}}(s):=\pi^{-s / 2} \Gamma(s / 2)$ and $\theta \in(-\pi / 2, \pi / 2)$ is a parameter. By [9, Sec. 6.699, Eqs. 3 and 4], we have

$$
\gamma_{\theta}(s, f)=4 i^{-\epsilon} w^{-1 / 2}\left(\frac{\cos \theta}{\sqrt{N}}\right)^{1 / 2-s} \frac{1}{\pi^{s+\epsilon}} \int_{0}^{\infty} K_{i r}(2 t) \frac{\cos ^{(-\epsilon)}(2 \tan (\theta) t)}{(2 \tan (\theta) t)^{\epsilon}} t^{s+\epsilon} \frac{d t}{t}
$$

for $\operatorname{Re} s>0$. (Note that for a Maass form with odd reflection symmetry, viz. $\epsilon=1,(2.2)$ has a removable singularity at $\theta=0$; this is related to the fact that the complete $L$-function is the Mellin transform of $\partial f / \partial x$ rather than $f$.) Making the substitution $t \mapsto \pi n \cos (\theta) u$, we can express the complete $L$-function $\Lambda_{\theta}(s, f):=\gamma_{\theta}(s, f) L(s, f)$ as the Mellin transform of the Maass form along a ray in the upper half plane:

$$
\begin{aligned}
\Lambda_{\theta}(s, f) & =\gamma_{\theta}(s, f) L(s, f) \\
& =c_{\theta}(s, f) \sum_{n=1}^{\infty} a_{n} \int_{0}^{\infty}(\cos (\theta) u)^{1 / 2} K_{i r}(2 \pi n \cos (\theta) u) \cos ^{(-\epsilon)}(2 \pi n \sin (\theta) u) u^{s-1 / 2} \frac{d u}{u} \\
& =c_{\theta}(s, f) \int_{0}^{\infty} f\left(i e^{i \theta} u\right) u^{s-1 / 2} \frac{d u}{u}, \quad \text { where } c_{\theta}(s, f):=\frac{4 w^{-1 / 2}}{(2 \pi i \tan \theta)^{\epsilon}} N^{\frac{1}{2}\left(s-\frac{1}{2}\right)} .
\end{aligned}
$$

Splitting the integral at $u=1 / \sqrt{N}$ and employing 2.1 completes the analytic continuation:

$$
\Lambda_{\theta}(s, f)=c_{\theta}(s, f)\left\{\int_{1 / \sqrt{N}}^{\infty} f\left(i e^{i \theta} u\right) u^{s-1 / 2} \frac{d u}{u}+\int_{0}^{1 / \sqrt{N}} \overline{w f\left(-\frac{1}{N i e^{i \theta} u}\right)} u^{s-1 / 2} \frac{d u}{u}\right\} .
$$

Using that $f(-\bar{z})=(-1)^{\epsilon} f(z)$ and making the substitution $u \mapsto \frac{1}{N u}$, we obtain the functional equation:

$$
\begin{aligned}
\Lambda_{\theta}(s, f) & =c_{\theta}(s, f) \int_{1 / \sqrt{N}}^{\infty} f\left(i e^{i \theta} u\right) u^{s-1 / 2} \frac{d u}{u}+\overline{c_{\theta}(1-\bar{s}, f)} \int_{1 / \sqrt{N}}^{\infty} \overline{f\left(i e^{i \theta} u\right)} u^{1 / 2-s} \frac{d u}{u} \\
& =\overline{\Lambda_{\theta}(1-\bar{s}, f) .}
\end{aligned}
$$

In particular, $\Lambda_{\theta}(1 / 2+i t, f) \in \mathbb{R}$ for $t \in \mathbb{R}$.

\section{Rigorous Computation of $L$-FunCtions}

We describe an algorithm based on the fast Fourier transform that allows one to evaluate $\Lambda_{\theta}(s, f)$ quickly, if one is interested in many points.

The integral 2.3 is essentially a Fourier transformation,

$$
\Lambda_{\theta}(\sigma+i t, f)=c_{\theta}(\sigma+i t, f) \int_{-\infty}^{\infty} f\left(i e^{i \theta} e^{u}\right) e^{u(\sigma-1 / 2)} e^{i u t} d u
$$

Similarly for the integral 2.2 ,

$$
\begin{aligned}
& \gamma_{\theta}(\sigma+i t, f) \\
& \quad=c_{\theta}(\sigma+i t, f) \int_{-\infty}^{\infty}\left(\cos (\theta) e^{u}\right)^{1 / 2} K_{i r}\left(2 \pi \cos (\theta) e^{u}\right) \cos ^{(-\epsilon)}\left(2 \pi \sin (\theta) e^{u}\right) e^{u(\sigma-1 / 2)} e^{i u t} d u
\end{aligned}
$$

In order to use the fast Fourier transform to compute

$$
g(t)=\int_{-\infty}^{\infty} \hat{g}(u) e^{i u t} d u
$$


we first need to discretize the integral. To that end, let $A, B>0$ be parameters such that $q=A B$ is an integer. In the Poisson summation formula,

$$
\sum_{k \in \mathbb{Z}} g\left(\frac{m}{A}+k B\right)=\frac{2 \pi}{B} \sum_{l \in \mathbb{Z}} e\left(\frac{m l}{q}\right) \hat{g}\left(\frac{2 \pi l}{B}\right),
$$

we solve for $g\left(\frac{m}{A}\right)$, which results in

$$
\begin{gathered}
g\left(\frac{m}{A}\right)=\frac{2 \pi}{B} \sum_{l=-C^{\prime}}^{C} e\left(\frac{m l}{q}\right) \hat{g}\left(\frac{2 \pi l}{B}\right)+\varepsilon_{g}, \\
\varepsilon_{g}:=\frac{2 \pi}{B} \sum_{\left\{l \in \mathbb{Z}: l<-C^{\prime} \text { or } l>C\right\}} e\left(\frac{m l}{q}\right) \hat{g}\left(\frac{2 \pi l}{B}\right)-\sum_{k \neq 0} g\left(\frac{m}{A}+k B\right) .
\end{gathered}
$$

In $\$$ we will derive precise bounds for this error term.

\section{Bounds}

Let $Q(s, f)$ be the analytic conductor, defined by

$$
Q(s, f):=N \frac{s+\epsilon+i r}{2 \pi} \frac{s+\epsilon-i r}{2 \pi} .
$$

Note that $\gamma(s, f)$ satisfies the recurrence $\gamma(s+2, f)=Q(s, f) \gamma(s, f)$. Further, we define

$$
\chi(s, f):=\frac{\overline{\gamma(1-\bar{s}, f)}}{\gamma(s, f)},
$$

so that $L(s, f)=\chi(s, f) \overline{L(1-\bar{s}, f)}$.

Lemma 4.1. 2, §4] For $s$ in the strip $\left\{s \in \mathbb{C}:-\frac{1}{2} \leq \operatorname{Re} s \leq \frac{3}{2}\right\}$,

$$
|L(s, f)|^{2} \leq|\chi(s, f) Q(s, f)| \sup _{t \in \mathbb{R}}\left|L\left(\frac{3}{2}+i t, f\right)\right|^{2} .
$$

Remark 4.2. The estimate in Lemma 4.1 is not optimal since, for $s=\frac{1}{2}+i t$ for large $t$, the right-hand side grows quadratically in $t$, whereas the convexity estimate would give $O\left(t^{1+\varepsilon}\right)$. Moreover, for $\epsilon=0$ and $s=1 \pm i r$ the bound becomes useless, since $|L(1 \pm i r, f)|<\infty$, whereas $\lim _{s \rightarrow 1 \pm i r}|\chi(s, f) Q(s, f)| \rightarrow \infty$. Nevertheless, the estimate is clean and uniform in all parameters, and suffices for our purposes if we keep away from $s=1 \pm i r$.

Corollary 4.3. For $s$ in the strip $\left\{s \in \mathbb{C}: \frac{1}{2} \leq \operatorname{Re} s<1\right\}$,

$$
|L(s, f)| \leq 3 N^{1 / 2}\left(|\operatorname{Im} s|+D_{s, f}\right)
$$

with

$$
D_{s, f}:=3 \operatorname{Re} s-1+\epsilon+|r|+\frac{(2 \operatorname{Re} s-1)^{2}}{1-\operatorname{Re} s+\epsilon} .
$$

Proof. Recall that $\Gamma_{\mathbb{R}}(s)$ satisfies the recurrence, reflection, and duplication formulas

$$
s \Gamma_{\mathbb{R}}(s)=2 \pi \Gamma_{\mathbb{R}}(2+s), \quad \Gamma_{\mathbb{R}}(s) \Gamma_{\mathbb{R}}(2-s)=\frac{1}{\sin \left(\frac{\pi}{2} s\right)}, \quad \text { and } \quad \Gamma_{\mathbb{R}}(s) \Gamma_{\mathbb{R}}(1+s)=2^{1-s} \Gamma_{\mathbb{R}}(2 s) .
$$

Hence, for $\epsilon \in\{0,1\}$ and $t \in \mathbb{R}$,

$$
\left|\frac{\Gamma_{\mathbb{R}}(2+\epsilon-i t)}{\Gamma_{\mathbb{R}}(1+\epsilon+i t)}\right|^{2}=\left\{\begin{array}{ll}
\frac{t^{2} \cosh \left(\frac{\pi}{2} t\right)}{2 \pi t \sinh \left(\frac{\pi}{2} t\right)} & \text { for } \epsilon=0 \\
\frac{\left(1+t^{2}\right) \sinh \left(\frac{\pi}{2} t\right)}{2 \pi t \cosh \left(\frac{\pi}{2} t\right)} & \text { for } \epsilon=1
\end{array}\right\} \leq \frac{(1+\epsilon)^{2}+t^{2}}{\pi^{2}},
$$

which yields

$$
|\chi(s, f) \overline{Q(1-\bar{s}, f)}| \leq 4 N^{-1 / 2}|Q(s, f)|
$$

for $\operatorname{Re} s=1$. 
On the critical line we have

$$
\left|\chi(s, f) \frac{\overline{Q(1-\bar{s}, f)}}{Q(s, f)}\right|_{\operatorname{Re} s=\frac{1}{2}}=1,
$$

and by the Phragmén-Lindelöf theorem,

$$
\sup _{\frac{1}{2} \leq \operatorname{Re} s \leq 1}\left|\chi(s, f) \frac{\overline{Q(1-\bar{s}, f)}}{Q(s, f)}\right| \leq \max \left\{4 N^{-1 / 2}, 1\right\} \leq 4 .
$$

Thus, in the strip $\frac{1}{2} \leq \operatorname{Re} s<1$ we have

$$
\left|\frac{Q(s, f)^{2}}{\overline{Q(1-\bar{s}, f)}}\right| \leq N\left(\frac{|\operatorname{Im} s|+D_{s, f}}{2 \pi}\right)^{2}
$$

Using the Kim-Sarnak estimate [1] $p^{-\vartheta} \leq\left|\alpha_{p}\right| \leq p^{\vartheta}$ with $\vartheta=\frac{7}{64}$ in the Euler product gives

$$
\sup _{\operatorname{Re} s=\frac{3}{2}}|L(s, f)|=\sup _{\operatorname{Re} s=\frac{3}{2}}\left|\prod_{p} \frac{1}{\left(1-\alpha_{p} p^{-s}\right)\left(1-\alpha_{p}^{-1} p^{-s}\right)}\right| \leq \zeta\left(\frac{3}{2}+\vartheta\right) \zeta\left(\frac{3}{2}-\vartheta\right)<3 \pi .
$$

Inserting the last three bounds in Lemma 4.1 yields the corollary.

Lemma 4.4. For $s=\sigma+i$ with $0<\sigma \leq 1$ and $0<\theta<\delta<\frac{\pi}{2}$,

$$
\left|\gamma_{\theta}(\sigma+i t, f)\right|<E_{\sigma, \theta, \delta} e^{-(\delta-\theta)|t|}
$$

with

$$
E_{\sigma, \theta, \delta}:=\frac{\left|c_{\theta}(\sigma, f)\right|}{(\cos (\delta-\theta))^{1 / 2}}\left\{\frac{\cosh (1) \sigma^{-1}\left(\sigma^{-1}+\log (2)+e^{-1}\right)}{(2 \pi)^{\sigma}(\cos (\delta-\theta) \cos \theta)^{\sigma-1 / 2}}+\frac{\Gamma\left(\sigma-\frac{1}{2}, \frac{\cos \delta}{\cos (\delta-\theta) \cos \theta}\right)}{2(2 \pi \cos \delta)^{\sigma-1 / 2}}\right\} .
$$

Proof. For $\gamma_{\theta}$ we have the integral representation $(2.2)$. Since $\left|\gamma_{\theta}(\sigma-i t, f)\right|=\left|\gamma_{\theta}(\sigma+i t, f)\right|$, it is enough to prove the lemma for non-negative $t$.

Making the change of variables $u \mapsto u+i(\delta-\theta)$ and moving the contour of integration back to the real line, we get

$$
g(t):=\gamma_{\theta}(\sigma+i t, f)=\int_{\mathbb{R}} \hat{g}(u) e^{i u t} d u=\int_{\mathbb{R}} \hat{g}(u+i(\delta-\theta)) e^{i(u+i(\delta-\theta)) t} d u,
$$

and

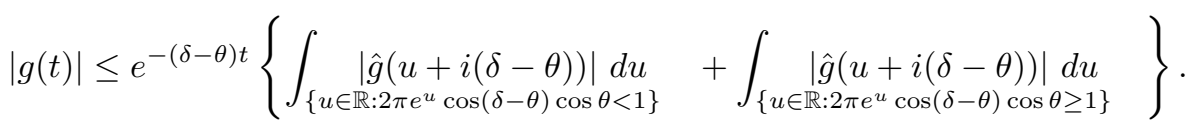

We bound the first integral using the estimates $\left|K_{i r}(z)\right| \leq \log \left(\frac{2}{\operatorname{Re} z}\right)+e^{-1}$ [3, p. 106] and $\left|\cos ^{(-\epsilon)}(\tan (\delta-\theta) \tan (\theta) \operatorname{Im} z)\right| \leq \cosh (1)$, and the second integral using $\left|K_{i r}(z)\right|<\left(\frac{\pi}{2 \operatorname{Re} z}\right)^{1 / 2} e^{-\operatorname{Re} z}$ and $\left|\cos ^{(-\epsilon)}(z)\right| \leq e^{|\operatorname{Im} z|}$.

Lemma 4.5. For $s=\sigma+i$ with $0<\sigma \leq 1,|t|=\frac{|m|}{A} \leq T \leq \frac{B}{2}$, and $0<\theta<\delta<\frac{\pi}{2}$,

$$
\left|\sum_{k \neq 0} \gamma_{\theta}\left(\sigma+i\left(\frac{m}{A}+k B\right), f\right)\right|<\frac{E_{\sigma, \theta, \delta}}{\sinh \left((\delta-\theta) \frac{B}{2}\right)} .
$$

Proof. Using Lemma 4.4 together with $\left|\frac{m}{A}+k B\right| \geq\left(|k|-\frac{1}{2}\right) B$ yields the stated bound.

Lemma 4.6. For $s=\sigma+i$ with $\frac{1}{2} \leq \sigma<1,|t|=\frac{|m|}{A} \leq T \leq \frac{B}{2}$, and $0<\theta<\delta<\frac{\pi}{2}$,

$$
\left|\sum_{k \neq 0} \Lambda_{\theta}\left(\sigma+i\left(\frac{m}{A}+k B\right), f\right)\right|<\frac{3 N^{1 / 2} E_{\sigma, \theta, \delta}}{\sinh \left((\delta-\theta) \frac{B}{2}\right)}\left(\frac{B}{2}+D_{\sigma, f}+\frac{B}{1-e^{-(\delta-\theta) B}}\right) .
$$


Proof. By Corollary 4.3 and Lemma 4.4 .

$$
\left|\Lambda_{\theta}(\sigma+i t, f)\right|<3 N^{1 / 2}\left(|t|+D_{\sigma, f}\right) E_{\sigma, \theta, \delta} e^{-(\delta-\theta)|t|} .
$$

Applying the estimate $\left(|k|-\frac{1}{2}\right) B \leq\left|\frac{m}{A}+k B\right| \leq\left(|k|+\frac{1}{2}\right) B$ and summing up results in the stated bound.

Lemma 4.7. For $\frac{1}{2} \leq \sigma \leq 1, B>0, C \geq \frac{B}{2 \pi} \log \frac{1+B /(2 \pi)}{4 \pi \cos \theta}, C^{\prime}=C+\frac{B}{2 \pi} \log N$, and $\hat{\Lambda}_{\sigma, \theta}(u, f):=$ $c_{\theta}(\sigma, f) f\left(i e^{i \theta} e^{u}\right) e^{u(\sigma-1 / 2)}$,

$$
\left|\frac{2 \pi}{B} \sum_{\left\{l \in \mathbb{Z}: l<-C^{\prime} \text { or } l>C\right\}} e\left(\frac{m l}{q}\right) \hat{\Lambda}_{\sigma, \theta}\left(\frac{2 \pi l}{B}, f\right)\right|<\frac{2 \pi}{B} \frac{56 N^{1 / 4}}{(2 \pi \tan \theta)^{\epsilon}} e^{\pi \frac{C}{B}-2 \pi \cos (\theta) e^{2 \pi \frac{C}{B}}} .
$$

Proof. Applying $\left|a_{n}\right| \leq 2 n^{1 / 2},\left|K_{i r}(y)\right|<\left(\frac{\pi}{2 y}\right)^{1 / 2} e^{-y}$, and $\left|\cos ^{(-\epsilon)}(x)\right| \leq 1$ in the Fourier expansion of the Maass form gives

$$
|f(x+i y)|<\frac{1}{e^{2 \pi y}-1},
$$

and by Fricke involution

$$
\left|f\left(i e^{i \theta} e^{u}\right)\right|=\left|\overline{f\left(i e^{-i \theta} e^{-u} / N\right)}\right|<\frac{1}{e^{2 \pi \cos (\theta) \max \left\{e^{u}, e^{-u} / N\right\}}-1} .
$$

For $\sigma \leq 1$,

$$
\left|\frac{2 \pi}{B} \sum_{l>C} e\left(\frac{m l}{q}\right) \hat{\Lambda}_{\sigma, \theta}\left(\frac{2 \pi l}{B}, f\right)\right|<\frac{2 \pi}{B} \sum_{l>C} \frac{4 N^{1 / 4}}{(2 \pi \tan \theta)^{\epsilon}} \frac{e^{\pi \frac{l}{B}}}{e^{2 \pi \cos (\theta) e^{2 \pi l / B}}-1} .
$$

Writing $a:=2 \pi \cos \theta, u:=2 \pi \frac{l}{B}, u_{0}:=2 \pi \frac{C}{B}$, with $a>0$ and $u_{0}<u$, we have

$$
\frac{e^{-a e^{u}+\frac{u}{2}}}{1-e^{-a e^{u}}}<\frac{e^{-a e^{u_{0}}\left(1+u-u_{0}\right)+\frac{u}{2}}}{1-e^{-a e^{u_{0}}}} .
$$

Summing the resulting geometric series gives

$$
\begin{aligned}
\left|\frac{2 \pi}{B} \sum_{l>C} e\left(\frac{m l}{q}\right) \hat{\Lambda}_{\sigma, \theta}\left(\frac{2 \pi l}{B}, f\right)\right| & <\frac{2 \pi}{B} \frac{4 N^{1 / 4}}{(2 \pi \tan \theta)^{\epsilon}} \frac{e^{-a e^{u_{0}}+\frac{u_{0}}{2}}}{\left(1-e^{-a e^{u_{0}}}\right)\left(1-e^{-\left(a e^{u_{0}}-\frac{1}{2}\right) \frac{2 \pi}{B}}\right)} \\
& <\frac{2 \pi}{B} \frac{4 N^{1 / 4}}{(2 \pi \tan \theta)^{\epsilon}} 7 e^{-a e^{u_{0}}+\frac{u_{0}}{2}}
\end{aligned}
$$

for $\frac{2 \pi}{B}\left(a e^{u_{0}}-\frac{1}{2}\right) \geq \frac{1}{2}$, and similarly for the sum over $l<-C$.

For $\frac{1}{2} \leq \sigma \leq 1$,

$$
\left|\frac{2 \pi}{B} \sum_{l<-C^{\prime}} e\left(\frac{m l}{q}\right) \hat{\Lambda}_{\sigma, \theta}\left(\frac{2 \pi l}{B}, f\right)\right|<\frac{2 \pi}{B} \sum_{l>C^{\prime}} \frac{4 N^{1 / 4}}{(2 \pi \tan \theta)^{\epsilon}} \frac{1}{e^{2 \pi \cos (\theta) e^{2 \pi l / B} / N}-1} .
$$

Writing $\frac{a}{N}:=\frac{2 \pi \cos \theta}{N}, u:=2 \pi \frac{|l|}{B}, u_{0}^{\prime}:=u_{0}+\log N=2 \pi \frac{C^{\prime}}{B}$, with $a>0$ and $u_{0}^{\prime}<u$, we have

$$
\frac{e^{-\frac{a}{N} e^{u}}}{1-e^{-\frac{a}{N} e^{u}}}<\frac{e^{-\frac{a}{N} e^{u_{0}^{\prime}}\left(1+u-u_{0}^{\prime}\right)}}{1-e^{-\frac{a}{N} e^{u_{0}^{\prime}}}}
$$

and summing up gives

$$
\left|\frac{2 \pi}{B} \sum_{l<-C^{\prime}} e\left(\frac{m l}{q}\right) \hat{\Lambda}_{\sigma, \theta}\left(\frac{2 \pi l}{B}, f\right)\right|<\frac{2 \pi}{B} \frac{4 N^{1 / 4}}{(2 \pi \tan \theta)^{\epsilon}} \frac{e^{-a e^{u_{0}}}}{\left(1-e^{-a e^{u_{0}}}\right)\left(1-e^{-a e^{u_{0}} \frac{2 \pi}{B}}\right)} .
$$


Lemma 4.8. For $\frac{1}{2} \leq \sigma \leq 1, B>0, C \geq \frac{B}{2 \pi} \log \frac{1+B /(2 \pi)}{4 \pi \cos \theta}, C^{\prime} \geq \frac{3 B}{2 \pi}$, and $\hat{\gamma}_{\sigma, \theta}(u, f):=c_{\theta}(\sigma, f)\left(\cos (\theta) e^{u}\right)^{1 / 2} K_{i r}\left(2 \pi \cos (\theta) e^{u}\right) \cos ^{(-\epsilon)}\left(2 \pi \sin (\theta) e^{u}\right) e^{u(\sigma-1 / 2)}$,

$$
\begin{aligned}
\left|\frac{2 \pi}{B} \sum_{\left\{l \in \mathbb{Z}: l<-C^{\prime} \text { or } l>C\right\}} e\left(\frac{m l}{q}\right) \hat{\gamma}_{\sigma, \theta}\left(\frac{2 \pi l}{B}, f\right)\right| \\
<\frac{2 \pi}{B} \frac{N^{1 / 4}}{(2 \pi \tan \theta)^{\epsilon}}\left\{6 e^{\pi \frac{C}{B}-2 \pi \cos (\theta) e^{2 \pi \frac{C}{B}}}+23(\cos \theta)^{1 / 6} \operatorname{sech}\left(\frac{\pi r}{2}\right) e^{-\frac{\pi C^{\prime}}{3 B}}\right\} .
\end{aligned}
$$

Proof. We have $\left|K_{i r}(y)\right|<\left(\frac{\pi}{2 y}\right)^{1 / 2} e^{-y}$ and $\left|\cos ^{(-\epsilon)}(x)\right| \leq 1$, so that

$$
\left|\frac{2 \pi}{B} \sum_{l>C} e\left(\frac{m l}{q}\right) \hat{\gamma}_{\sigma, \theta}\left(\frac{2 \pi l}{B}, f\right)\right|<\frac{2 \pi}{B} \sum_{l>C} \frac{4 N^{1 / 4}}{(2 \pi \tan \theta)^{\epsilon}} 2^{-1} e^{-2 \pi \cos (\theta) e^{2 \pi \frac{l}{B}}} e^{\pi \frac{l}{B}} .
$$

Writing $a:=2 \pi \cos \theta, u:=2 \pi \frac{l}{B}, u_{0}:=2 \pi \frac{C}{B}$, with $a>0$ and $u_{0}<u$, we have

$$
e^{-a e^{u}+\frac{u}{2}}<e^{-a e^{u_{0}}\left(1+u-u_{0}\right)+\frac{u}{2}}
$$

and summing the geometric series yields

$$
\begin{aligned}
\left|\frac{2 \pi}{B} \sum_{l>C} e\left(\frac{m l}{q}\right) \hat{\gamma}_{\sigma, \theta}\left(\frac{2 \pi l}{B}, f\right)\right| & <\frac{2 \pi}{B} \frac{4 N^{1 / 4}}{(2 \pi \tan \theta)} 2^{-1} \frac{e^{-a e^{u_{0}}+\frac{u_{0}}{2}}}{1-e^{-\left(a e^{u}-\frac{1}{2}\right) \frac{2 \pi}{B}}} \\
& <\frac{2 \pi}{B} \frac{6 N^{1 / 4}}{(2 \pi \tan \theta)^{\epsilon}} e^{-a e^{u_{0}}+\frac{u_{0}}{2}}
\end{aligned}
$$

for $\frac{2 \pi}{B}\left(a e^{u_{0}}-\frac{1}{2}\right) \geq \frac{1}{2}$.

The argument is slightly different for the sum over $l<-C$. Using that $\left|\cosh \left(\frac{\pi r}{2}\right) K_{i r}(y)\right|<$ $4 y^{-1 / 3}$ [3, p. 107] and $\left|\cos ^{(-\epsilon)}(x)\right| \leq 1$, we have

$$
\begin{aligned}
\left|\frac{2 \pi}{B} \sum_{l<-C^{\prime}} e\left(\frac{m l}{q}\right) \hat{\gamma}_{\sigma, \theta}\left(\frac{2 \pi l}{B}, f\right)\right| & <\frac{2 \pi}{B} \sum_{l>C^{\prime}} \frac{4 N^{1 / 4}}{(2 \pi \tan \theta)^{\epsilon}} \frac{4(2 \pi)^{-1 / 3}}{\cosh \left(\frac{\pi r}{2}\right)}\left(\cos (\theta) e^{-\frac{2 \pi|l|}{B}}\right)^{1 / 6} \\
& <\frac{2 \pi}{B} \frac{23 N^{1 / 4}}{(2 \pi \tan \theta)^{\epsilon}}(\cos \theta)^{1 / 6} \operatorname{sech}\left(\frac{\pi r}{2}\right) e^{-\frac{\pi C^{\prime}}{3 B}}
\end{aligned}
$$

for $\frac{\pi C^{\prime}}{3 B} \geq \frac{1}{2}$.

The following two Lemmata show that we can circumvent division by zero in $L(s, f)=\frac{\Lambda_{\theta}(s, f)}{\gamma_{\theta}(s, f)}$.

Lemma 4.9. For any $y \in \mathbb{R}$ with $|y|>5,\left|\Gamma_{\mathbb{R}}(x+i y)\right|$ is an increasing function of $x \in \mathbb{R}$.

Proof. It suffices to show that $\frac{\partial}{\partial x} \log \left|\Gamma_{\mathbb{R}}(x+i y)\right|=\operatorname{Re} \psi_{\mathbb{R}}(x+i y)>0$. Suppose first that $x \geq 1$. Then, differentiating Binet's formula for $\log \Gamma_{\mathbb{R}}$, we have

$$
\begin{aligned}
\operatorname{Re} \psi_{\mathbb{R}}(x+i y) & =\frac{1}{2} \log \left(x^{2}+y^{2}\right)-\frac{x}{x^{2}+y^{2}}-\frac{1}{2} \log (4 \pi)-\int_{0}^{\infty}\left(1-\frac{1}{t}+\frac{2}{e^{2 t}-1}\right) e^{-x t} \cos (y t) d t \\
& \geq \frac{1}{2} \log \left(x^{2}+y^{2}\right)-\frac{x}{x^{2}+y^{2}}+\psi_{\mathbb{R}}(1)+1 .
\end{aligned}
$$

For $x \geq 1$, this is easily seen to be minimum at $x=1$, so we obtain

$$
\operatorname{Re} \psi_{\mathbb{R}}(x+i y) \geq \frac{1}{2} \log \left(1+y^{2}\right)-\frac{1}{1+y^{2}}+\psi_{\mathbb{R}}(1)+1 .
$$

For $x \leq 1$ we use the reflection formula $\psi_{\mathbb{R}}(z)=\psi_{\mathbb{R}}(2-z)-\pi \cot \left(\frac{\pi}{2} z\right)$ to see that

$$
\operatorname{Re} \psi_{\mathbb{R}}(x+i y)=\operatorname{Re} \psi_{\mathbb{R}}(2-(x+i y))-\operatorname{Re} \pi \cot \left(\frac{\pi}{2}(x+i y)\right),
$$

and apply the above to obtain a bound for $\operatorname{Re} \psi_{\mathbb{R}}(2-(x+i y))$. We calculate that

$$
\operatorname{Re} \cot \left(\frac{\pi}{2}(x+i y)\right)=\frac{2 e^{\pi y} \sin (\pi x)}{1-2 e^{\pi y} \cos (\pi x)+e^{2 \pi y}},
$$


and with a little calculus we see that this is bounded in modulus by $1 / \sinh (\pi|y|)$. Thus, altogether we have

$$
\operatorname{Re} \psi_{\mathbb{R}}(x+i y) \geq \frac{1}{2} \log \left(1+y^{2}\right)-\frac{1}{1+y^{2}}-\frac{\pi}{\sinh (\pi|y|)}+\psi_{\mathbb{R}}(1)+1
$$

for all $x \in \mathbb{R}$ and $y \neq 0$. Note that the right-hand side is strictly increasing for $y>0$. Using the known value $\psi_{\mathbb{R}}(1)=-\gamma-\frac{1}{2} \log (16 \pi)=-2.53587 \ldots$, it is straightforward to see that $\operatorname{Re} \psi_{\mathbb{R}}(x+i y)$ is positive for $|y|>5$.

Lemma 4.10. For $r>5, \frac{1}{2} \leq \sigma \leq 1,0<\theta_{1}<\theta_{2}<\frac{\pi}{2}, \cos \theta_{1} \leq\left(4+\left|t^{2}-r^{2}\right|\right)^{-1 / 2}$, and $\cos \theta_{2}=e^{-\frac{\pi}{2 r}} \cos \theta_{1}$,

$$
\max \left|\gamma_{\theta_{1}}(s, f), \gamma_{\theta_{2}}(s, f)\right| \geq \frac{2}{3}\left(\cos \theta_{2}\right)^{\frac{1}{2}+\epsilon}(2 \pi)^{-\epsilon}\left(\frac{\pi}{r \sinh (\pi r)}\right)^{1 / 2} .
$$

Proof. We follow the proof of [17, generalizing it and making the implied constants explicit. Using [9. Sec. 9.132, Eq. 1] and writing

$$
g_{\theta}(s, f):=\Gamma_{\mathbb{R}}(s+\epsilon+i r) \frac{\Gamma_{\mathbb{R}}(-2 i r) \Gamma_{\mathbb{R}}(1+2 \epsilon)}{\Gamma_{\mathbb{R}}(1-s+\epsilon-i r)}{ }_{2} F_{1}\left(\frac{s+\epsilon+i r}{2}, \frac{1-s+\epsilon+i r}{2} ; i r+1 ; \cos ^{2} \theta\right),
$$

we get

$$
\gamma_{\theta}(s, f)=i^{-\epsilon} w^{-1 / 2} N^{\frac{1}{2}\left(s-\frac{1}{2}\right)}\left\{(\cos \theta)^{\frac{1}{2}+\epsilon+i r} g_{\theta}(s, f)+(\cos \theta)^{\frac{1}{2}+\epsilon-i r} \overline{g_{\theta}(\bar{s}, f)}\right\} .
$$

By Lemma 4.9 for $\sigma \geq \frac{1}{2}$ and $|t+r|>5$,

$$
\left|\frac{\Gamma_{\mathbb{R}}(s+\epsilon+i r)}{\Gamma_{\mathbb{R}}(1-s+\epsilon-i r)}\right|=\left|\frac{\Gamma_{\mathbb{R}}(2 \sigma-1+1-\sigma+\epsilon+i(t+r))}{\Gamma_{\mathbb{R}}(1-\sigma+\epsilon-i(t+r))}\right| \geq 1 .
$$

Next,

$$
\left|\Gamma_{\mathbb{R}}(-2 i r) \Gamma_{\mathbb{R}}(1+2 \epsilon)\right|=(2 \pi)^{-\epsilon}\left(\frac{\pi}{r \sinh (\pi r)}\right)^{1 / 2},
$$

and for $\frac{1}{2} \leq \sigma \leq 1$,

$$
\left|\frac{\left(\frac{s+\epsilon+i r}{2}+n\right)\left(\frac{1-s+\epsilon+i r}{2}+n\right)}{(i r+1+n)(1+n)}\right|<1+\frac{\left|t^{2}-r^{2}\right|}{4} .
$$

Hence for $\cos ^{2} \theta \leq\left(4+\left|t^{2}-r^{2}\right|\right)^{-1}$,

$$
{ }_{2} F_{1}\left(\frac{s+\epsilon+i r}{2}, \frac{1-s+\epsilon+i r}{2} ; i r+1 ; \cos ^{2} \theta\right)=1+\sum_{n=1}^{\infty} \Theta\left(\frac{1}{4^{n}}\right)=1+\Theta\left(\frac{1 / 4}{1-1 / 4}\right),
$$

where $\Theta(x)$ stands for a value of absolute size at most $x$.

$r>5$ implies that $|t+r|>5$ or $|t-r|>5$. Thus

$$
\max \left|g_{\theta}(s, f), \overline{g_{\theta}(\bar{s}, f)}\right| \geq(2 \pi)^{-\epsilon}\left(\frac{\pi}{r \sinh (\pi r)}\right)^{1 / 2}\left(1-\Theta\left(\frac{1}{3}\right)\right) .
$$

Adjusting the phase factor $(\cos \theta)^{i r}$ in (4.3) suitably, i.e. taking $\theta=\theta_{1}$ and $\theta=\theta_{2}$ with $\left(\cos \theta_{2}\right)^{2 i r}=$ $e^{-i \pi}\left(\cos \theta_{1}\right)^{2 i r}$, respectively, completes the proof.

\section{INTERPOLATING ZEROS}

We compute values of $L$ on a grid, but we are ultimately interested in the zeros, which are not regularly spaced. To zoom in on the zeros, we interpolate

$$
h(t):=e^{-\frac{\left(t-t_{0}\right)^{2}}{2 b^{2}}} g(t)
$$


with $g(t)=\Lambda_{\theta}(\sigma+i t, f)$ and $g(t)=\gamma_{\theta}(\sigma+i t, f)$, respectively. The function $h$ has the advantage that it decays rapidly at $\infty$ and is approximately bandlimited, which allows us to use the Whittaker-Shannon sampling theorem [5]

$$
\left|h(t)-\sum_{m \in \mathbb{Z}} h\left(\frac{m}{A}\right) \operatorname{sinc}\left(\pi A\left(t-\frac{m}{A}\right)\right)\right| \leq 4 \int_{\pi A}^{\infty}|\hat{h}(u)| d u .
$$

Truncating the sum over $m$ and bounding the error of truncation, we get an effective interpolation formula for $h$.

Lemma 5.1. For $g(t):=\Lambda_{\theta}(\sigma+i t, f), G:=\max _{|t| \leq T}|g(t)|, \frac{1}{A} \leq J \leq T-\left|t_{0}\right|$, and $b>0$,

$$
\begin{aligned}
& \left|\sum_{\left|\frac{m}{A}-t_{0}\right|>J} h\left(\frac{m}{A}\right) \operatorname{sinc}\left(\pi A\left(t-\frac{m}{A}\right)\right)\right|<\sqrt{2 \pi} b A\left\{G \operatorname{erfc}\left(\frac{J-\frac{1}{A}}{\sqrt{2} b}\right)\right. \\
& \left.+3 N^{1 / 2} E_{\sigma, \theta, \delta} e^{-(\delta-\theta) T}\left\{\frac{2 b}{\sqrt{2 \pi}} e^{-\frac{\left(T-\left|t_{0}\right|-\frac{1}{A}\right)^{2}}{2 b^{2}}}+\left(\left|t_{0}\right|+D_{\sigma, f}\right) \operatorname{erfc}\left(\frac{T-\left|t_{0}\right|-\frac{1}{A}}{\sqrt{2} b}\right)\right\}\right\} .
\end{aligned}
$$

Proof. For $|t| \leq T$, we have $|g(t)| \leq G$, and for $|t|>T$, we bound $|g(t)|$ by 4.1. Hence,

$$
\begin{aligned}
& \left|\sum_{\left|\frac{m}{A}-t_{0}\right|>J} h\left(\frac{m}{A}\right) \operatorname{sinc}\left(\pi A\left(t-\frac{m}{A}\right)\right)\right| \leq \sum_{\left|\frac{m}{A}-t_{0}\right|>J} e^{-\frac{\left(\frac{m}{A}-t_{0}\right)^{2}}{2 b^{2}}}\left|g\left(\frac{m}{A}\right)\right| \\
& <\sum_{\substack{\left|\frac{m}{A}-t_{0}\right|>J \\
\left|\frac{m}{A}\right| \leq T}} e^{-\frac{\left(\frac{m}{A}-t_{0}\right)^{2}}{2 b^{2}}} G+\sum_{\substack{\left|\frac{m}{A}-t_{0}\right|>J \\
\left|\frac{m}{A}\right|>T}} e^{-\frac{\left(\frac{m}{A}-t_{0}\right)^{2}}{2 b^{2}}} 3 N^{1 / 2}\left(\left|\frac{m}{A}\right|+D_{\sigma, f}\right) E_{\sigma, \theta, \delta} e^{-(\delta-\theta)\left|\frac{m}{A}\right|} \\
& <G \int_{\left|\frac{m}{A}-t_{0}\right|>J-\frac{1}{A}} e^{-\frac{\left(\frac{m}{A}-t_{0}\right)^{2}}{2 b^{2}}} d m+3 N^{1 / 2} E_{\sigma, \theta, \delta} e^{-(\delta-\theta) T} \int_{\left|\frac{m}{A}\right|>T-\frac{1}{A}} e^{-\frac{\left(\frac{m}{A}-t_{0}\right)^{2}}{2 b^{2}}}\left(\left|\frac{m}{A}\right|+D_{\sigma, f}\right) d m .
\end{aligned}
$$

Lemma 5.2. For $g(t):=\gamma_{\theta}(\sigma+i t, f), G:=\max _{|t| \leq T}|g(t)|, \frac{1}{A} \leq J \leq T-\left|t_{0}\right|$, and $b>0$,

$$
\begin{array}{r}
\left|\sum_{\left|\frac{m}{A}-t_{0}\right|>J} h\left(\frac{m}{A}\right) \operatorname{sinc}\left(\pi A\left(t-\frac{m}{A}\right)\right)\right|<\sqrt{2 \pi} b A\left\{G \operatorname{erfc}\left(\frac{J-\frac{1}{A}}{\sqrt{2} b}\right)\right. \\
\left.+E_{\sigma, \theta, \delta} e^{-(\delta-\theta) T} \operatorname{erfc}\left(\frac{T-\left|t_{0}\right|-\frac{1}{A}}{\sqrt{2} b}\right)\right\} .
\end{array}
$$

Proof. The proof is similar to that of the previous lemma, except that for $|t|>T$ we bound $|g(t)|$ by Lemma 4.4 .

Lemma 5.3. For $g(t):=\Lambda_{\theta}(\sigma+i t, f), \frac{1}{2} \leq \sigma \leq 1, u_{0} \geq 0, \pi A \geq u_{0}$, and $b>0$,

$$
\begin{aligned}
4 \int_{\pi A}^{\infty}|\hat{h}(u)| d u<2\left|c_{\theta}(\sigma, f)\right| \frac{e^{u_{0}\left(\sigma-\frac{1}{2}\right)}}{1-e^{-2 \pi \cos (\theta) / \sqrt{N}}}\left\{\frac{\sqrt{2 \pi}}{2 b} \operatorname{erfc}\left(\frac{b}{\sqrt{2}}\left(\pi A-u_{0}\right)\right)\right. \\
\left.+\frac{2 e^{-u_{0}\left(\sigma-\frac{1}{2}\right)}}{(2 \pi \cos \theta)^{\sigma-\frac{1}{2}}} \Gamma\left(\sigma-\frac{1}{2}, 2 \pi \cos (\theta) e^{u_{0}}\right) .\right\}
\end{aligned}
$$

Proof. By Fourier convolution,

$$
\hat{h}(v)=\frac{b}{\sqrt{2 \pi}} \int_{\mathbb{R}} e^{-\frac{b^{2}}{2}(v-u)^{2}-i(v-u) t_{0}} \hat{g}(u) d u
$$


with $\hat{g}(u):=c_{\theta}(\sigma, f) f\left(i e^{i \theta} e^{u}\right) e^{u\left(\sigma-\frac{1}{2}\right)}$, as defined in 2.3 $)$. Using 4.2 and writing $a:=2 \pi \cos \theta$ gives

$$
|\hat{h}(v)|<\frac{b}{\sqrt{2 \pi}} \int_{\mathbb{R}} e^{-\frac{b^{2}}{2}(v-u)^{2}} \frac{\left|c_{\theta}(\sigma, f)\right| e^{u\left(\sigma-\frac{1}{2}\right)}}{e^{a \max \left\{e^{u}, e^{-u} / N\right\}}-1} d u .
$$

Changing the order of integration, we have

$$
\begin{aligned}
4 \int_{\pi A}^{\infty}|\hat{h}(v)| d v & <\frac{4 b}{\sqrt{2 \pi}}\left|c_{\theta}(\sigma, f)\right| \int_{\mathbb{R}} \frac{d u e^{u\left(\sigma-\frac{1}{2}\right)}}{e^{a \max \left\{e^{u}, e^{-u} / N\right\}}-1} \int_{\pi A}^{\infty} d v e^{-\frac{b^{2}}{2}(v-u)^{2}} \\
& =2\left|c_{\theta}(\sigma, f)\right| \int_{\mathbb{R}} \frac{e^{u\left(\sigma-\frac{1}{2}\right)} \operatorname{erfc}\left(\frac{b}{\sqrt{2}}(\pi A-u)\right)}{e^{a \max \left\{e^{u}, e^{-u} / N\right\}}-1} d u
\end{aligned}
$$

Let $u_{0} \in[0, \pi A]$ and set $x:=\frac{b}{\sqrt{2}}(\pi A-u)$. For $u<u_{0}$, we have $x>0$ and $\operatorname{erfc}(x)<e^{-x^{2}}$, while for $u \geq u_{0}, \operatorname{erfc}(x)<2$. Moreover, for $u<u_{0}, a>0, \sigma \geq \frac{1}{2}$,

$$
\frac{e^{u\left(\sigma-\frac{1}{2}\right)}}{e^{a \max \left\{e^{u}, e^{-u} / N\right\}}-1}<\frac{e^{u_{0}\left(\sigma-\frac{1}{2}\right)}}{1-e^{-a / \sqrt{N}}}
$$

while for $u \geq u_{0}$,

$$
\frac{1}{e^{a \max \left\{e^{u}, e^{-u} / N\right\}}-1}<\frac{1}{\left(1-e^{-a / \sqrt{N}}\right) e^{a e^{u}}}
$$

Thus,

$$
4 \int_{\pi A}^{\infty}|\hat{h}(v)| d v<2\left|c_{\theta}(\sigma, f)\right| \frac{e^{u_{0}\left(\sigma-\frac{1}{2}\right)}}{1-e^{-a / \sqrt{N}}}\left\{\int_{-\infty}^{u_{0}} e^{-\frac{b^{2}}{2}(\pi A-u)^{2}} d u+2 e^{-u_{0}\left(\sigma-\frac{1}{2}\right)} \int_{u_{0}}^{\infty} \frac{e^{u\left(\sigma-\frac{1}{2}\right)}}{e^{a e^{u}}} d u\right\} .
$$

Identifying the integrals with the complementary error function and the incomplete gamma function completes the proof.

Lemma 5.4. For $g(t):=\gamma_{\theta}(\sigma+i t, f), \frac{1}{2} \leq \sigma \leq 1, u_{0} \geq 0, \pi A \geq u_{0}$, and $b>0$,

$$
\begin{aligned}
4 \int_{\pi A}^{\infty}|\hat{h}(u)| d u<2\left|c_{\theta}(\sigma, f)\right|\left\{\frac{2}{b} e^{u_{0}\left(\sigma-\frac{1}{2}\right)}\left(2 \pi \cos (\theta) e^{u_{0}}\right)^{1 / 6} \operatorname{sech}\left(\frac{\pi r}{2}\right) \operatorname{erfc}\left(\frac{b}{\sqrt{2}}\left(\pi A-u_{0}\right)\right)\right. \\
\left.+(2 \pi \cos \theta)^{\frac{1}{2}-\sigma} \Gamma\left(\sigma-\frac{1}{2}, 2 \pi \cos (\theta) e^{u_{0}}\right)\right\} .
\end{aligned}
$$

Proof. By Fourier convolution,

$$
\hat{h}(v)=\frac{b}{\sqrt{2 \pi}} \int_{\mathbb{R}} e^{-\frac{b^{2}}{2}(v-u)^{2}-i(v-u) t_{0}} \hat{g}(u) d u,
$$

with $\hat{g}(u):=c_{\theta}(\sigma, f)\left(\cos (\theta) e^{u}\right)^{1 / 2} K_{i r}\left(2 \pi \cos (\theta) e^{u}\right) \cos ^{(-\epsilon)}\left(2 \pi \sin (\theta) e^{u}\right) e^{u(\sigma-1 / 2)}$, as in 2.2 a). For $u<u_{0}$, we have $\left|\cosh \left(\frac{\pi r}{2}\right) K_{i r}(y)\right|<4 y^{-1 / 3}$ [3, p. 107], $\left|\cos ^{(-\epsilon)}(x)\right| \leq 1$, and $e^{u\left(\sigma-\frac{1}{2}\right)}<e^{u_{0}\left(\sigma-\frac{1}{2}\right)}$. Hence, writing $\frac{a}{2 \pi}:=\cos \theta>0$, we have

$$
\int_{-\infty}^{u_{0}} e^{-\frac{b^{2}}{2}(v-u)^{2}}|\hat{g}(u)| d u<\left|c_{\theta}(\sigma, f)\right| e^{u_{0}\left(\sigma-\frac{1}{2}\right)} \int_{-\infty}^{u_{0}} e^{-\frac{b^{2}}{2}(v-u)^{2}} \frac{4(2 \pi)^{-1 / 3}}{\cosh \left(\frac{\pi r}{2}\right)}\left(\frac{a}{2 \pi} e^{u}\right)^{1 / 6} d u .
$$

For $u \geq u_{0} \geq 0$, we use $\left|K_{i r}(y)\right|<\left(\frac{\pi}{2 y}\right)^{1 / 2} e^{-y}$, and $\left|\cos ^{(-\epsilon)}(x)\right| \leq 1$ to obtain

$$
\int_{u_{0}}^{\infty} e^{-\frac{b^{2}}{2}(v-u)^{2}}|\hat{g}(u)| d u<\left|c_{\theta}(\sigma, f)\right| \int_{u_{0}}^{\infty} e^{-\frac{b^{2}}{2}(v-u)^{2}} \frac{1}{2} e^{-a e^{u}} e^{u\left(\sigma-\frac{1}{2}\right)} d u
$$




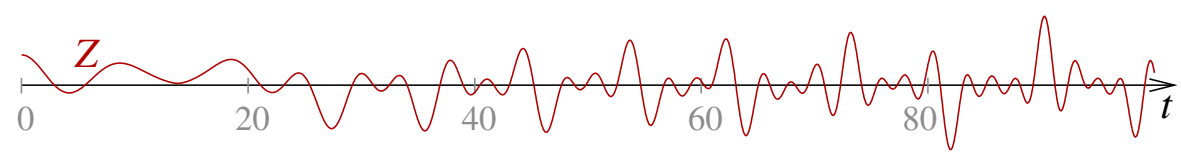

Figure 1. Graph of $Z(t, f)$ for the first even Maass form $L$-function on $\operatorname{SL}(2, \mathbb{Z})$.

Therefore, after interchanging the order of integration,

$$
\begin{aligned}
& 4 \int_{\pi A}^{\infty}|h(v)| d v<\frac{4 b}{\sqrt{2 \pi}}\left|c_{\theta}(\sigma, f)\right|\left\{e^{u_{0}\left(\sigma-\frac{1}{2}\right)} \int_{-\infty}^{u_{0}} \frac{4(2 \pi)^{-1 / 2}}{\cosh \left(\frac{\pi r}{2}\right)}\left(a e^{u}\right)^{1 / 6} \int_{\pi A}^{\infty} e^{-\frac{b^{2}}{2}(v-u)^{2}} d v d u\right. \\
& \left.+\int_{u_{0}}^{\infty} \frac{1}{2} e^{-a e^{u}} e^{u\left(\sigma-\frac{1}{2}\right)} \int_{\pi A}^{\infty} e^{-\frac{b^{2}}{2}(v-u)^{2}} d v d u\right\} \\
& <2\left|c_{\theta}(\sigma, f)\right|\left\{e^{u_{0}\left(\sigma-\frac{1}{2}\right)} \frac{4(2 \pi)^{-1 / 2}}{\cosh \left(\frac{\pi r}{2}\right)}\left(a e^{u_{0}}\right)^{1 / 6} \int_{-\infty}^{u_{0}} e^{-\frac{b^{2}}{2}(\pi A-u)^{2}} d u+\int_{u_{0}}^{\infty} e^{-a e^{u}} e^{u\left(\sigma-\frac{1}{2}\right)} d u\right\},
\end{aligned}
$$

where we have employed $\operatorname{erfc}(x) \leq e^{-x^{2}}$ for $x \geq 0$, and $\operatorname{erfc}(x)<2$ otherwise. Evaluating the integrals completes the proof.

\section{Detecting Zeros}

For each Maass form $L$-function under consideration, we compute rigorously many values on the critical line. For instance, Figure 1 shows a graph of

$$
Z(t, f):=L\left(\frac{1}{2}+i t, f\right) e^{i \arg \gamma\left(\frac{1}{2}+i t, f\right)}
$$

for the first even Maass form $L$-function on $\Gamma(1)$.

Corollary 6.1. Let $\Omega_{\gamma_{\theta}}:=\left\{t \in \mathbb{R}: \gamma_{\theta}\left(\frac{1}{2}+i t\right)=0\right\}$ be the set of zeros on the critical line of the $\Gamma$-factor. For values of $\theta_{1}$ and $\theta_{2}$ chosen according to Lemma 4.10 .

$$
\Omega_{\gamma_{\theta_{1}}} \cap \Omega_{\gamma_{\theta_{2}}}=\emptyset \text {. }
$$

Proof. This follows immediately from Lemma 4.10 .

Remark 6.2. Fixing the value of $\theta$, say $\theta=\theta_{1}$, there is a risk of hitting a zero of $\gamma_{\theta_{1}}$ (to within the internal precision) when evaluating $Z_{\theta_{1}}$ for some specific value of $t$. In all of our computations, we never observed this in practice, i.e. we never had to deal with division by zero. However, computing at finite absolute precision, we sometimes come close to a zero of $\gamma_{\theta_{1}}$ and experience some loss of precision in the division by $\left|\gamma_{\theta_{1}}\right|$. Since we also compute for a second value of $\theta$, $\theta=\theta_{2}$, chosen according to Lemma 4.10, we may always ensure the accuracy of the computed values of $Z$.

For each Maass form $L$-function under consideration, we rigorously compute all zeros on the critical line up to some height. The search for zeros is faciliated by the following lemma.

Lemma 6.3. (a) Let $Z \in C^{1}(\mathbb{R})$ be real valued, and assume it has consecutive simple zeros at $t_{0}$, $t_{1}$ and $t_{2}$, with $Z^{\prime}\left(t_{0}\right)>0$. Then $\exists t_{a}, t_{b}, t_{c}, t_{d}, t_{e}, t_{f}$ such that the following holds:

\begin{tabular}{c|ccc}
$t$ & $Z^{\prime}(t)$ & $Z(t)$ & quadrant of $Z^{\prime}+i Z$ \\
\hline$t_{a}<t<t_{0}$ & $>0$ & $<0$ & 4 \\
$t_{0}<t<t_{b}$ & $>0$ & $>0$ & 1 \\
$t_{b} \leq t \leq t_{c}$ & & $>0$ & 1 or 2 \\
$t_{c}<t<t_{1}$ & $<0$ & $>0$ & 2 \\
$t_{1}<t<t_{d}$ & $<0$ & $<0$ & 3 \\
$t_{d} \leq t \leq t_{e}$ & & $<0$ & 3 or 4 \\
$t_{e}<t<t_{2}$ & $>0$ & $<0$ & 4 \\
$t_{2}<t<t_{f}$ & $>0$ & $>0$ & 1
\end{tabular}


(b) Let $Z \in C^{n}(\mathbb{R})$ be real valued, and assume it has a zero of order $n$ at $t_{0}$, with $\left(\frac{d^{n}}{d t^{n}} Z\right)\left(t_{0}\right)>0$. Then $\exists t_{a}, t_{b}$ such that the following holds:

\begin{tabular}{c|cccc}
$t$ & $\frac{d^{n}}{d t^{n}} Z$ & $\frac{d^{n-1}}{d t^{n-1}} Z$ & $\frac{d^{n-2 k}}{d t^{n-2 k}} Z$ & $\frac{d^{n-2 k-1}}{d t^{n-2 k-1}} Z$ \\
\hline$t_{a}<t<t_{0}$ & $>0$ & $<0$ & $>0$ & $<0$ \\
$t=t_{0}$ & $>0$ & $=0$ & $=0$ & $=0$ \\
$t_{0}<t<t_{b}$ & $>0$ & $>0$ & $>0$ & $>0$
\end{tabular}

for $k \in \mathbb{Z}$, but $0<2 k<n$.

Proof. The lemma follows from elementary analysis and the intermediate value theorem.

Algorithm 6.4. Let $Z \in C^{\infty}(\mathbb{R})$ be real valued. Let $\left(t_{j}\right)_{j \in \mathbb{N}}$ be a strictly increasing sequence of real numbers. Refine the sequence $\left(t_{j}\right)$ until all zeros of $Z$ are isolated, i.e. there is at most one zero per interval $\left(t_{j}, t_{j+1}\right]$.

Remark 6.5. If the quadrants of $Z^{\prime}+i Z$ for consecutive $t_{j}$ are not ordered as given in Lemma 6.3(a), there is either a zero of order greater than 1 which is to be investigated according to Lemma $6.3(\mathrm{~b})$, or the sequence is not yet fine enough. We expect the sequence to be fine enough if for successive $t_{j}$ the quadrants of $Z^{\prime}+i Z$ do change by at most by 1 , and when they change, they do so in agreement with Lemma 6.3 .

There is no proof that the expectation in Remark 6.5 holds, and one can construct sequences $\left(t_{j}\right)$ that contradict the expectation. Nevertheless, with some reasonable choices in the construction of the sequence $\left(t_{j}\right)$ and its refinements, the expectation turns out to be reliable in practice. Namely, for every Maass form $L$-function that we considered, we never overlooked any zero, as proven after the fact using Turing's method.

\section{TURING'S METHOD}

Turing's method for verifying the Riemann hypothesis for arbitrary $L$-functions is described in [2]. For $t$ not the ordinate of some zero or pole of $\Lambda$, let

$$
S(t):=\frac{1}{\pi} \operatorname{Im} \int_{\infty}^{\frac{1}{2}} \frac{L^{\prime}}{L}(\sigma+i t, f) d \sigma .
$$

By convention, we make $S(t)$ upper semicontinuous, i.e. when $t$ is the ordinate of a zero or pole, we define $S(t)=\lim _{\varepsilon \rightarrow 0^{+}} S(t+\varepsilon)$.

We select a particular branch of $\log \gamma(s)$ by using the principal branch of $\log \Gamma$. With this choice, set

We further define

$$
\Phi(t):=\frac{1}{\pi} \operatorname{Im} \log \gamma\left(\frac{1}{2}+i t, f\right) .
$$

$$
N(t):=\Phi(t)+S(t),
$$

which relates to the number of zeros in the critical strip up to height $t$. For $t_{1}<t_{2}$ let $\Omega_{L}$ denote the multiset of zeros with imaginary part in $\left(t_{1}, t_{2}\right]$, and let $N\left(t_{1}, t_{2}\right)$ denote their number, counting multiplicity,

Then, we have

$$
N\left(t_{1}, t_{2}\right):=\# \Omega_{L}\left(t_{1}, t_{2}\right) .
$$

$$
N\left(t_{1}, t_{2}\right)=N\left(t_{2}\right)-N\left(t_{1}\right) .
$$

Theorem 7.1. [2, §4] For $\vartheta=\frac{7}{64}$ and $\sigma>\vartheta+1$, define

$$
z_{\vartheta}(\sigma):=\left(\frac{\zeta(2 \sigma+2 \vartheta) \zeta(2 \sigma-2 \vartheta)}{\zeta(\sigma+\vartheta) \zeta(\sigma-\vartheta)}\right)^{1 / 2}
$$

and

Suppose $t_{1}$ and $t_{2}$ satisfy

$$
Z_{\vartheta}(\sigma):=(\zeta(\sigma+\vartheta) \zeta(\sigma-\vartheta))^{1 / 2} .
$$

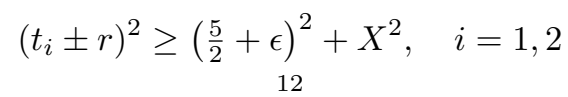


for some $X>5$, and set

$$
C_{\vartheta}:=\log Z_{\vartheta}\left(\frac{3}{2}\right)+\int_{3 / 2}^{\infty} \log \frac{Z_{\vartheta}(\sigma)}{z_{\vartheta}(\sigma)} d \sigma-\int_{3 / 2}^{5 / 2} \log z_{\vartheta}(\sigma) d \sigma+(\log 4) \frac{z_{\vartheta}^{\prime}\left(\frac{3}{2}\right)}{z_{\vartheta}\left(\frac{3}{2}\right)} .
$$

Then

$$
\pi \int_{t_{1}}^{t_{2}} S(t) d t \leq \frac{1}{4} \log \left|Q\left(\frac{3}{2}+i t_{2}\right)\right|+\left(\log 2-\frac{1}{2}\right) \log \left|Q\left(\frac{3}{2}+i t_{1}\right)\right|+2 C_{\vartheta}+\frac{2}{\sqrt{2}(X-5)} .
$$

Corollary 7.2. For $0 \leq t_{1}<t_{2}$, assume $\tilde{\Omega}_{L}\left(t_{1}, t_{2}\right)$ is a given multiset of zeros with imaginary part in $\left(t_{1}, t_{2}\right]$, i.e. $\tilde{\Omega}_{L}\left(t_{1}, t_{2}\right) \subseteq \Omega_{L}\left(t_{1}, t_{2}\right)$. Let

$$
\begin{array}{ll} 
& N_{\tilde{\Omega}_{L}}\left(t_{1}, t_{2}\right):=\# \tilde{\Omega}_{L}\left(t_{1}, t_{2}\right), \quad \text { counting multiplicity, } \\
& N_{\tilde{\Omega}_{L}}(t):=N_{\tilde{\Omega}_{L}}(t, 0)+\Phi(0)+S(0), \\
\text { and } \quad & S_{\tilde{\Omega}_{L}}(t):=N_{\tilde{\Omega}_{L}}(t)-\Phi(t) .
\end{array}
$$

If

$$
\pi \int_{t_{1}}^{t_{2}}\left(S_{\tilde{\Omega}_{L}}(t)+1\right) d t
$$

exceeds the right-hand side of the bound in Theorem 7.1. then the set $\tilde{\Omega}_{L}\left(0, t_{1}\right)$ contains all zeros with imaginary part in $\left(0, t_{1}\right] . \tilde{\Omega}_{L}\left(0, t_{1}\right)=\Omega_{L}\left(0, t_{1}\right)$.

Proof. If $\tilde{\Omega}_{L}\left(0, t_{1}\right)$ were a proper subset of $\Omega_{L}\left(0, t_{1}\right)$, then we would have $N_{\tilde{\Omega}_{L}}\left(t_{1}\right)<N\left(t_{1}\right)$, whence $S_{\tilde{\Omega}_{L}}(t)+1 \leq S(t) \forall t \in\left(t_{1}, t_{2}\right]$. But the integral of the latter is bounded by Theorem 7.1 .

\section{NUMERICAL RESUlts}

We consider consecutive Maass cusp forms on $\operatorname{SL}(2, \mathbb{Z})=\Gamma(1)$. Booker, Strömbergsson, and Venkatesh 4 have rigorously computed the first 10 Maass cusp forms on $\operatorname{SL}(2, \mathbb{Z})$ to high precision. Bian [1] has extended these computations to a larger number of Maass cusp forms. The readily available list of rigorously computed Maass cusp forms is consecutive for the first 2191 Maass cusp forms, which covers all Maass cusp forms whose Laplacian eigenvalue $\lambda=r^{2}+\frac{1}{4}$ falls into the range $0 \leq r \leq 178$.

Previous numerical computations of some non-trivial zeros for a few even Maass form $L$ functions were made by Strömbergsson [18. We extend his results by rigorously computing, for each of the first 2191 consecutive Maass form $L$-functions on $\mathrm{SL}(2, \mathbb{Z})$, many values of $Z$, including all non-trivial zeros up to $T=30000$, at least.

Remark 8.1. At the time of Strömbergsson's work, even the numerical data pertaining to the Maass cusp forms for $\mathrm{SL}(2, \mathbb{Z})$ was not rigorously proven to be accurate, so he had no reason to carry out his computations of the zeros with more than heuristic estimates for the error. Making use of the rigorous data sets described above, we have rigorously verified the correctness of Strömbergsson's results. In particular his lists of zeros are consecutive and accurate. Moreover, we confirm his observation of a zero-free region on the critical line for $t$ near $r$, when $r$ is small.

We note that some theoretical results, such as Cho's theorem [6] on simple zero of Maass form $L$-functions, assumed the correctness of Strömbergsson's numerical results. With our verification, Cho's theorem becomes unconditional.

Our lists of zeros contain more than 60000 consecutive non-trivial zeros per Maass form $L$ function. All these zeros are simple. The first several zeros of the first five Maass form $L$-functions are listed in Table 1

Theorem 8.2. For $f$ a Maass cusp form on $\mathrm{SL}(2, \mathbb{Z})$ with spectral parameter $0 \leq r \leq 178$, all non-trivial zeros with $0 \leq t \leq 30000$ of the corresponding Maass form L-function are simple and on the critical line.

Proof. For each Maass form $L$-function we prove, using Corollary 7.2 , that the corresponding list of rigorously computed zeros is consecutive for $0 \leq t \leq 30000$, and that all the zeros are indeed simple and on the critical line. 
TABLE 1. Consecutive lists of the first few non-trivial zeros for the first five Maass form $L$-functions on $\mathrm{SL}(2, \mathbb{Z})$. Each column is for one Maass form $L$-function and is specified by the spectral parameter $r$ and the parity $\epsilon$. The displayed numbers are the ordinates of the first few consecutive zeros for $t>0$, all of which are on the critical line. Each number is accurate to within \pm 1 in the last digit.

\begin{tabular}{|c|c|c|c|c|}
\hline $\begin{array}{c}r=9.533695261 \\
\epsilon=1\end{array}$ & $\begin{array}{c}r=12.173008325 \\
\epsilon=1\end{array}$ & $\begin{array}{c}r=13.779751352 \\
\epsilon=0\end{array}$ & $\begin{array}{c}r=14.358509518 \\
\epsilon=1\end{array}$ & $\begin{array}{c}r=16.138073172 \\
\epsilon=1\end{array}$ \\
\hline 17.0249420759926 & 5.10553130864728 & 2.89772467827094 & 3.76470190452593 & 4.07043016260804 \\
\hline 19.3441154991815 & 17.7442287880043 & 5.59124531531950 & 8.44187034414965 & 6.01471804932679 \\
\hline 22.8261931283343 & 22.0828833772350 & 21.0903775087339 & 19.448354 & 896 \\
\hline 25.7999235601013 & 00118314732 & 45853 & 1538 & 34970933 \\
\hline 27.6164361749163 & 7.1426126160360 & 8372 & 26.1518661201714 & 23.6756749096999 \\
\hline 29.1018834648622 & 8.8988378613334 & 9.1892067135368 & 27.8260578322407 & 27.7899319219294 \\
\hline 31.8310613699717 & 1.2199778278305 & 1.0617394845440 & 30.9903075480748 & 30.0381347329908 \\
\hline 34.3471038793177 & 3.3027699993553 & 32.4527182375570 & 32.0681458350820 & 32.0229736589354 \\
\hline 35.6095026712633 & 1.9413315016281 & 34.0272796838472 & 35.0463081449147 & 33.8112506234903 \\
\hline 00794665599 & & & חקדת תמ & 02 \\
\hline & & & & \\
\hline & & & & \\
\hline 42.9624682023 & & & & 98 \\
\hline & & & 04 & 81 \\
\hline & & & & \\
\hline & & & 69 & 75 \\
\hline & & & & \\
\hline & & & & \\
\hline 52 . & & 26 & 81 & \\
\hline & & & & \\
\hline 54.8 & & & 762 & 262 \\
\hline 56.1642766726 & 774 & 72 & 3777 & 301 \\
\hline 57.747715804 & & & & \\
\hline 59.1886 & & & & \\
\hline & & & & \\
\hline & & & & \\
\hline & & & & \\
\hline & & & & \\
\hline & & & & \\
\hline & & & & \\
\hline & & & & \\
\hline & & & & \\
\hline & & & & \\
\hline 72.7 & & & & \\
\hline & & & & \\
\hline & & & & \\
\hline 76.6 & & & & \\
\hline 77 . & 5 & & & \\
\hline 79.517782453 & & & & \\
\hline & & & & \\
\hline & & & & \\
\hline & & & & \\
\hline & & & & \\
\hline & & & & \\
\hline & & & & \\
\hline & & & & \\
\hline & & & & 57 \\
\hline & & & & \\
\hline & & & & \\
\hline & & & & 88 \\
\hline & & & & 775 \\
\hline & & & & 870 \\
\hline & & & 3961 & 95.7412 \\
\hline & & & & 622 \\
\hline 98.11595683 & 534 & & 7.99687 & 97.4404 \\
\hline & & 46 & .2448 & 236 \\
\hline & & & & \\
\hline & & & & \\
\hline & & & & \\
\hline & & & & \\
\hline & & & & \\
\hline 106.296 & & & 22 & 23 \\
\hline & & & & 106.554 \\
\hline & & & & 977 \\
\hline & & & & 3655 \\
\hline & & & & \\
\hline & & 71 & 18 & 33 \\
\hline 112.822069704440 & 112.96824433 & 11245294741 & 112.6773 & 112.76 \\
\hline 113.6421667 & & 51 & 3 & 879 \\
\hline 114.945311219697 & 114.803248583420 & 114.5994 & 114.342832613189 & 114.136514740415 \\
\hline
\end{tabular}




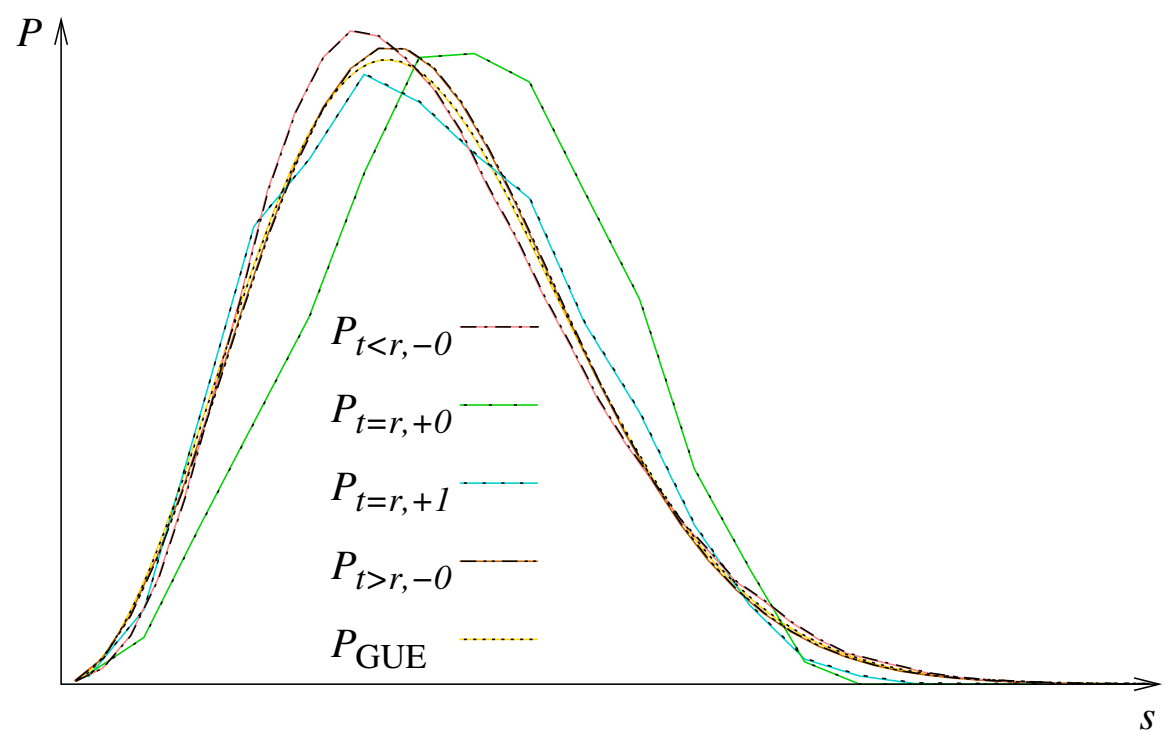

Figure 2. Rescaled nearest-neighbor spacing distributions $P_{t<r,-0}, P_{t=r,+0}$, $P_{t=r,+1}$, and $P_{t>r,-0}$, respectively, in comparison with the Wigner surmise $P_{\mathrm{GUE}}$. Only the distribution of zeros that are in absolute size closest to the value of the spectral parameter might show a stronger level repulsion than the Wigner surmise. In all other cases, the distribution of zeros closely resembles GUE statistics.

According to a conjecture of Montgomery [14, the distribution of non-trivial zeros should follow random matrix theory (RMT) predictions. In case of Maass form $L$-functions, the distribution of non-trivial zeros is expected to conform to that of eigenvalues of large random matrices from the Gaussian unitary ensemble (GUE) [10]. This raises the question of how GUE statistics relate to the zero-free region around $t=r$ observed by Strömbergsson [18] - are the GUE statistics asymptotically correct in the large $t$ aspect only?

We investigate this question by distinguishing between zeros with small and large absolute ordinate, respectively. For a given Maass form $L$-function there are only a finite number of zeros with small ordinate, and the resulting statistics would be poor. Knowing the zeros for many Maass form $L$-functions, we can evaluate on a common scale the distribution of zeros for each $L$-function and collate the statistics of many of them together.

Let $f$ be a Maass cusp form with spectral parameter $r$ and parity $\epsilon$. Consider the zeros of the associated Maass form $L$-function. We unfold the zeros,

$$
x_{i}:=\Phi\left(t_{i}\right),
$$

in order to obtain rescaled zeros $x_{i}$ with a unit mean density. Then $s_{i}:=x_{i+i}-x_{i}$ defines the sequence of nearest-neighbor spacings, which has mean value 1 as $i \rightarrow \infty$. Now, the distribution of nearest-neighbor spacings is given by

$$
\int_{0}^{s} P_{f}\left(s^{\prime}\right) d s^{\prime}:=\lim _{j \rightarrow \infty} \frac{\#\left\{i \leq j: s_{i} \leq s\right\}}{\#\{i \leq j\}},
$$

where the index $f$ denotes the corresponding Maass cusp form. Distributions of rescaled nearestneighbor spacings are expected to be independent of the specific parameter values of corresponding Maass cusp forms $f$ and can be collated by writing

$$
P(s):=\frac{1}{\#\{f\}} \sum_{f} P_{f}(s) .
$$




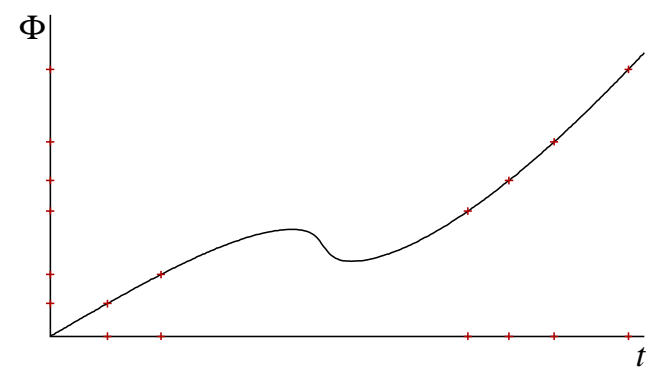

Figure 3. Average number $\Phi$ of non-trivial zeros for the first even Maass form $L$-function on $\mathrm{SL}(2, Z)$. For comparison, the locations of zeros and unfolded zeros are also included as tics. Clearly visible is the negative density region $\left(\Phi^{\prime}<0\right)$ for $t$ around $r$. Zeros are pulled away from this region resulting in a zero-free region in the $t$ aspect, but not in the unfolded zeros.

To distinguish between zeros with small and large absolute ordinate, we define the respective nearest-neighbor spacings distributions,

$$
\begin{aligned}
\int_{0}^{s} P_{f, t<r,-n}\left(s^{\prime}\right) d s^{\prime} & :=\lim _{j \rightarrow \infty} \frac{\#\left\{i \leq j: s_{i} \leq s, 0<t_{i-1}, t_{i+n-1}<r\right\}}{\#\left\{i \leq j: 0<t_{i-1}, t_{i+n-1}<r\right\}}, \\
\int_{0}^{s} P_{f, t=r,+n}\left(s^{\prime}\right) d s^{\prime} & :=\lim _{j \rightarrow \infty} \frac{\#\left\{i \leq j: s_{i} \leq s, t_{i-1-n}<r<t_{i+n}\right\}}{\#\left\{i \leq j: t_{i-1-n}<r<t_{i+n}\right\}}, \\
\int_{0}^{s} P_{f, t>r,-n}\left(s^{\prime}\right) d s^{\prime} & :=\lim _{j \rightarrow \infty} \frac{\#\left\{i \leq j: s_{i} \leq s, t_{i-n}>r\right\}}{\#\left\{i \leq j: t_{i-n}>r\right\}},
\end{aligned}
$$

where $n$ is a non-negative integer, as well as their collated versions

$$
\begin{aligned}
& P_{t<r,-n}(s):=\frac{1}{\#\{f\}} \sum_{f} P_{f, t<r,-n}(s), \\
& P_{t=r,+n}(s):=\frac{1}{\#\{f\}} \sum_{f} P_{f, t=r,+n}(s), \\
& P_{t>r,-n}(s):=\frac{1}{\#\{f\}} \sum_{f} P_{f, t>r,-n}(s) .
\end{aligned}
$$

For the first 2191 Maass form $L$-functions on $\operatorname{SL}(2, \mathbb{Z})$, the resulting distributions are displayed in Figure 2, in comparison with the Wigner surmise

$$
P_{\mathrm{GUE}}(s):=\frac{32}{\pi^{2}} s^{2} e^{-\frac{4 s^{2}}{\pi}} .
$$

As is visible, the distribution of zeros resembles GUE statistics for both small and large absolute ordinate, and there appears to be no distinction between the statistics of the two cases. Only the distribution of zeros that are in absolute size closest to the value of the spectral parameter might show a stronger level repulsion than the Wigner surmize.

However, it is unclear whether this seemingly stronger level repulsion is just an artefact of the limited number of 2191 spacings that contribute to the histogram of $P_{t=r,+0}$. If we take three times as many spacings into account, as is the case with $P_{t=r,+1}$, we again find a close resemblance to the Poisson distribution. We speculate that the GUE statistics hold for all $t$, not only in the large $t$ aspect.

Since the GUE statistics are based on rescaled zeros, $x_{i}=\Phi\left(t_{i}\right)$, they do not contradict a zero-free region on the critical line. The density of zeros is described by $\Phi^{\prime}$, and according to the $\Gamma$-factor, the density of zeros is smaller for $t$ in a neighborhood of $r$. In particular, for small values of the spectral parameter $r$, the density $\Phi^{\prime}$ becomes negative for $t$ near $r$; see Figure 3 . There are finitely many Maass form $L$-functions on $\mathrm{SL}(2, \mathbb{Z})$ that have such a region where $\Phi^{\prime}$ is negative. 


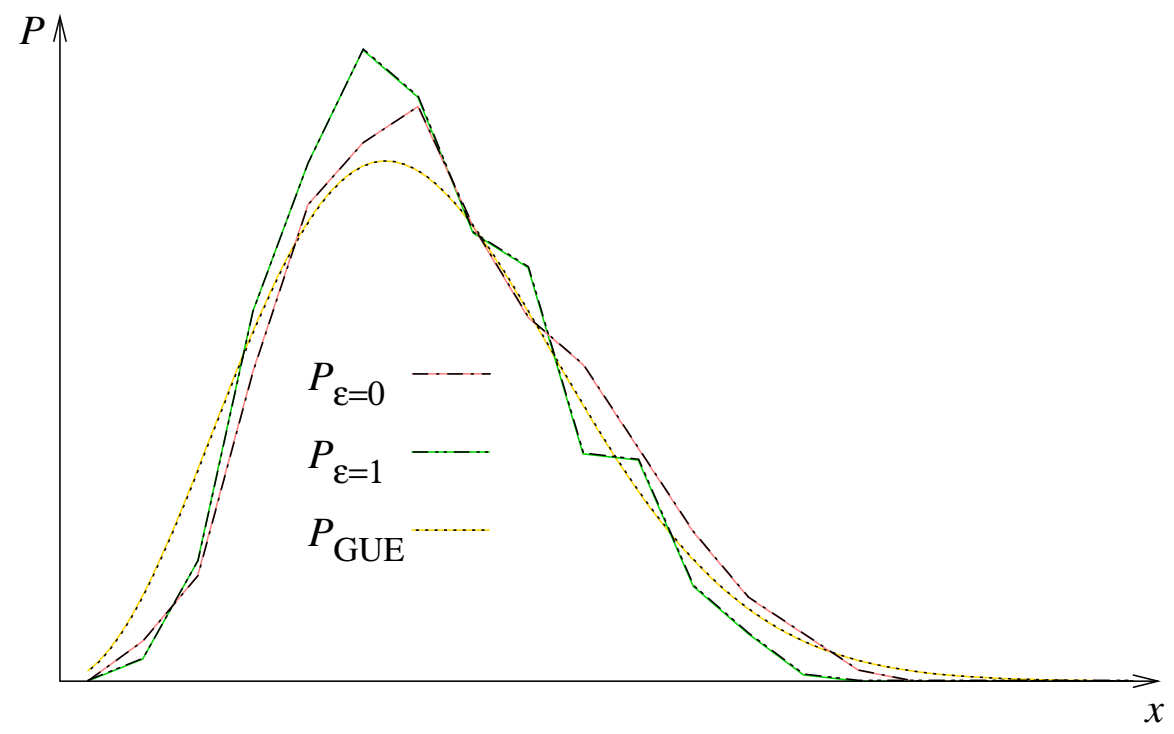

FiguRE 4. Distributions of the rescaled first zero in dependence of the parity of the Maass form $L$-function. Amongst the 2191 Maass cusp forms under consideration, 1018 of them are even with respect to reflection in the imaginary axis, $\epsilon=0$, and 1173 of them are odd, $\epsilon=1$. In comparison with the Wigner surmise $P_{\mathrm{GUE}}$, close to the origin of the plots, the first zero shows a stronger level repulsion irrespective of the parity.

By inspection, we find that no zero falls into a negative density region. Moreover, the zeros seem to be repelled away from the negative density regions resulting in the zero-free region around $r$.

Finally, we investigate the repulsion from zero of the rescaled first zero $x_{1}$ in dependence of the parity $\epsilon$ of the Maass form $L$-function. For this we consider the distributions of the rescaled first zero,

$$
\int_{0}^{x} P_{\epsilon=e}\left(x^{\prime}\right) d x^{\prime}:=\lim _{r \rightarrow \infty} \frac{\#\left\{j: x_{1}<x, r_{j} \leq r, \epsilon=e\right\}}{\#\left\{j: r_{j} \leq r, \epsilon=e\right\}},
$$

for $e \in\{0,1\}$. The resulting distributions are displayed in Figure 4 . Close to the origin of the plots, they show a stronger level repulsion than the Wigner surmise $P_{\mathrm{GUE}}(x)$.

\section{REFERENCES}

[1] C. Bian, A. R. Booker, and M. Jacobson, Unconditional computation of the class groups of real quadratic fields, in preparation.

[2] A. R. Booker, Artin's conjecture, Turing's method, and the Riemann hypothesis, Experiment. Math. 15 (2006), $385-408$.

[3] A. R. Booker, A. Strömbergsson, and H. Then, Bounds and algorithms for the K-Bessel function of imaginary order, LMS J. Comp. Math. 16 (2013), 78-108.

[4] A. R. Booker, A. Strömbergsson, and A. Venkatesh, Effective computation of Maass cusp forms, Int. Math. Res. Notices 2006 (2006), article ID 71281.

[5] J. L. Brown Jr., On the error in reconstructing a non-bandlimited function by means of the bandpass sampling theorem, J. Math. Anal. Appl. 18 (1967), 75-84.

[6] P. J. Cho, Simple zeros of Maass $L$-functions, Int. J. Number Theory 9 (2013), 167-178.

[7] T. Dokchitser, Computing special values of motivic $L$-functions, Experiment. Math. 13 (2004), 137-149.

[8] A. Good, On various means involving the Fourier coefficients of cusp forms, Math. Z. 183 (1983), 95-129.

[9] I. S. Gradshteyn and I. M. Ryzhik, Table of Integrals, Series, and Products, Academic Press, 2007.

[10] J. P. Keating and N. C. Snaith, Random matrix theory and $\zeta(1 / 2+i t)$, Commun. Math. Phys. 214 (2000), $57-89$.

[11] H. H. Kim and P. Sarnak, Refined estimates towards the Ramanujan and Selberg conjectures, J. Amer. Math. Soc. 16 (2003), 139-183.

[12] J. C. Lagarias and A. M. Odlyzko, On computing Artin $L$-functions in the critical strip, Math. Comp. 33 (1979), 1081-1095. 
[13] P. Molin, Intégration numérique et calculs de fonctions L. PhD thesis, Université Bordeaux I, 2010.

[14] H. L. Montgomery, The pair correlation of zeros of the zeta function. Analytic number theory (Proc. Sympos. Pure Math. 24, St. Louis Univ., St. Louis, Mo., 1972), pp. 181-193. Amer. Math. Soc., Providence, R.I., 1973.

[15] MPFI, multiple precision floating-point interval library, http://perso.ens-lyon.fr/nathalie.revol/ software.html

[16] M. Rubinstein, Computational methods and experiments in analytic number theory, In Recent Perspectives in Random Matrix Theory and Number Theory, pp. 425-506, London Math. Soc. Lecture Note Ser. 322, Cambridge, Cambridge Univ. Press, 2005.

[17] P. Sarnak, Fourth moments of grossencharakteren zeta functions, Comm. Pure and Appl. Math. 38 (1985), $167-178$

[18] A. Strömbergsson, On the zeros of $L$-functions associated to Maass waveforms, Int. Math. Res. Notices 1999 (1999), 839-851.

[19] P. Vishe, Rapid computation of L-functions for modular forms, Int. Math. Res. Notices (2012), doi:10.1093/imrn/rns112

Department of Mathematics, University of Bristol, University Walk, Bristol, BS8 1TW, United KINGDOM, E-MAIL: ANDREW.BOOKER@BRISTOL.AC.UK

Alemannenweg 1, 89537 Giengen, Germany, E-mail: Holger.then@Bristol.AC.uk 